\title{
Particle-number projected electric quadrupole moment of even-even proton-rich nuclei in the isovector pairing case
}

\author{
Mohamed Douici ${ }^{1,2}$, Nassima-Hosni Allal ${ }^{1,3 a}$, Mohamed Fellah ${ }^{1,3}$, Naziha Benhamouda $^{1}$ and \\ Mohamed-Reda Oudih ${ }^{1}$ \\ ${ }^{1}$ Laboratoire de Physique Théorique, Faculté de Physique, USTHB, BP32 El-Alia, 16111 Bab- \\ Ezzouar, Alger, Algeria \\ ${ }^{2}$ Institut des Sciences et Technologie, Centre Universitaire de Khemis Miliana, Route de Theniet-El- \\ Had, 44225 Khemis-Miliana, Algeria \\ ${ }^{3}$ Centre de Recherche Nucléaire d'Alger, COMENA, BP399 Alger-Gare, Alger, Algeria
}

\begin{abstract}
The effect of the particle-number projection on the electric quadrupole moment $\left(\mathrm{Q}_{2}\right)$ of even-even proton-rich nuclei is studied in the isovector neutron-proton (np) pairing case. As a first step, an expression of the electric quadrupole moment, which takes into account the isovector np pairing effect and which conserves the particlenumber, is established within the Sharp-BCS (SBCS) method. This expression does generalize the one used in the pairing between like-particles case. As a second step, $\mathrm{Q}_{2}$ is calculated for even-even proton-rich nuclei using the single-particle energies of a WoodsSaxon mean-field. The obtained results are compared with the results obtained in the pairing between like-particles case. It is shown that the np pairing effect, as well as the projection one, is maximal when $\mathrm{N}=\mathrm{Z}$.
\end{abstract}

\section{Introduction}

The study of the properties of exotic nuclei is one of the most active areas in nuclear physics. Since the advent of radioactive beams, it is possible to study nuclei far from the valley of stability and in particular proton-rich nuclei [1,2]. The latter are important for both nuclear physics and astrophysics. Pairing is an important component of nuclear structure studies. However, it is now well established that in proton-rich nuclei, the neutron-proton (np) pairing is no more negligible [3]. Indeed, in this kind of nuclei, identical single-particle orbitals are available for both the protons and the neutrons. This fact allows the creation of np pairs.

On the other hand, the electric quadrupole moment is of particular interest since it is an excellent tool to get information about the deformation of nuclei [4]. Many studies have been devoted to this observable either on the experimental side or the theoretical one. However, most of the theoretical works deal with ordinary or neutron-rich nuclei. Thus, when the pairing correlations are taken into account, it is only in the pairing between like-particles case, even if a projection method is used [5,6]. As for studies dealing with proton-rich nuclei, they don't take into account the np pairing correlations.

The aim of the present contribution is to study the effect of the isovector $\mathrm{np}$ pairing and the particle-number projection on the electric quadrupole moment of some even-even proton-rich nuclei

\footnotetext{
${ }^{a}$ Corresponding author: nallal@usthb.dz; allaln@yahoo.com
}

This is an Open Access article distributed under the terms of the Creative Commons Attribution License 2.0, which permits unrestricted use, distribution, and reproduction in any medium, provided the original work is properly cited. 
using the Sharp-BCS (SBCS) method. The latter is a generalization of the one used in the pairing between like-particles case $[5,6]$.

\section{Formalism}

Let us consider a system of $\mathrm{N}=2 \mathrm{P}_{\mathrm{n}}$ neutrons and $\mathrm{Z}=2 \mathrm{P}_{\mathrm{p}}$ protons. The neutrons and the protons are assumed to occupy the same energy levels. The total Hamiltonian of the system is given, in the isovector case, by [7-10]:

$$
\hat{\mathrm{H}}=\sum_{v>0, t} \varepsilon_{v t}\left(\mathrm{a}_{v \mathrm{t}}^{+} \mathrm{a}_{v \mathrm{t}}+\mathrm{a}_{\tilde{v} \mathrm{v}}^{+} \mathrm{a}_{\tilde{v} \mathrm{t}}\right)-\frac{1}{2} \sum_{\mathrm{tt}} \mathrm{G}_{\mathrm{tt}} \sum_{v, \mu>0}\left(\mathrm{a}_{v \mathrm{t}}^{+} \mathrm{a}_{\tilde{v} \mathrm{t}^{\prime}}^{+} \mathrm{a}_{\tilde{\mu} \mathrm{t}^{\prime}} \mathrm{a}_{\mu \mathrm{t}}+\mathrm{a}_{v \mathrm{t}}^{+} \mathrm{a}_{\tilde{v} \mathrm{t}^{+}}^{+} \mathrm{a}_{\tilde{\mu} \mathrm{t}} \mathrm{a}_{\mu \mathrm{t}^{\prime}}\right)
$$

where $t$ corresponds to the isospin component $(t=n, p), a_{v t}^{+}$and $a_{v t}$ respectively represent the creation and annihilation operators of the particle in the state $|v t\rangle$, of energy $\varepsilon_{v t} ;|\tilde{v} t\rangle$ is the time-reverse of $|v t\rangle$ and has the same energy. $G_{t t}$ characterizes the pairing-strength. The use of the linearization method, within the BCS approach, leads to the generalized Bogoliubov-Valatin transformation. $\hat{\mathrm{H}}$ then reads in the quasiparticle (qp) representation and in the framework of the independent qp approximation:

$$
\hat{\mathrm{H}}=\mathrm{E}_{0}+\sum_{v>0, \tau, \tau^{\prime}} \mathrm{E}_{v \tau \tau^{\prime}}\left(\alpha_{v \tau}^{+} \alpha_{v \tau^{\prime}}+\alpha_{\tilde{v} \tau}^{+} \alpha_{\tilde{v} \tau^{\prime}}\right)
$$

where $\alpha_{v \tau}^{+}\left(\alpha_{v \tau}\right)$ is the creation (annihilation) operator of a qp of energy $E_{v \tau \tau}$ and $\tau$ is the qp type $(\tau=1,2)$. As the Hamiltonian (2) is still non-diagonal, one has to carry out a rediagonalization, using the method described in Ref. [8]. $\hat{H}$ then becomes :

$$
\hat{\mathrm{H}}=\mathrm{E}_{0}+\sum_{v>0, \tau} \chi_{v \tau}\left(\beta_{v \tau}^{+} \beta_{v \tau}+\beta_{\tilde{v} \tau}^{+} \beta_{\tilde{v} \tau}\right)
$$

$\beta_{v \tau}^{+}$being the new qp operator and $\chi_{v \tau}$ the new qp energy.

The BCS state is the qp vacuum and is given by:

$$
|\psi\rangle=\prod_{v>0}\left|\psi_{v}\right\rangle,\left|\psi_{v}\right\rangle=\left[\mathrm{B}_{1}^{v} \mathrm{~A}_{v \mathrm{n}}^{+} \mathrm{A}_{v \mathrm{p}}^{+}+\mathrm{B}_{\mathrm{p}}^{v} \mathrm{~A}_{v \mathrm{p}}^{+}+\mathrm{B}_{\mathrm{n}}^{v} \mathrm{~A}_{v \mathrm{n}}^{+}+\mathrm{B}_{4}^{v}\left(\mathrm{a}_{\tilde{v} \mathrm{n}}^{+} \mathrm{a}_{v \mathrm{p}}^{+}+\mathrm{a}_{\tilde{v} \mathrm{p}}^{+} \mathrm{a}_{v \mathrm{n}}^{+}\right)+\mathrm{B}_{5}^{v}\right]|0\rangle
$$

where $\mathrm{A}_{v \mathrm{t}}^{+}=\mathrm{a}_{\tilde{v} \mathrm{t}}^{+} \mathrm{a}_{v \mathrm{t}}^{+} \mathrm{t}=\mathrm{n}, \mathrm{p}$, and $\mathrm{B}_{\mathrm{i}}^{v}, \mathrm{i}=1, \mathrm{p}, \mathrm{n}, 4,5$ are the various occupation probabilities.

However, the state (4) is not an eigen-state of the particle-number operator. It is thus projected using the method described in Refs. [7-10]. The corresponding wave-function is given by:

$$
\left|\psi_{\mathrm{mm}^{\prime}}\right\rangle=\mathrm{C}_{\mathrm{mm}^{\prime}}\left\{\sum_{\mathrm{k}=0}^{\mathrm{m}+1} \sum_{\mathrm{k}^{\prime}=0}^{\mathrm{m}^{\prime}+1} \xi_{\mathrm{k}} \xi_{\mathrm{k}^{\prime}}\left[\mathrm{z}_{\mathrm{k}}^{-\mathrm{P}_{\mathrm{n}}} \mathrm{z}_{\mathrm{k}}^{-\mathrm{P}_{\mathrm{p}}}\left|\psi\left(\mathrm{z}_{\mathrm{k}}, \mathrm{z}_{\mathrm{k}^{\prime}}\right)\right\rangle+\overline{\mathrm{z}}_{\mathrm{k}}^{-\mathrm{P}_{\mathrm{n}}} \mathrm{z}_{\mathrm{k}}^{-\mathrm{P}_{\mathrm{p}}} \psi\left|\left(\overline{\mathrm{z}}_{\mathrm{k}}, \mathrm{z}_{\mathrm{k}^{\prime}}\right)\right\rangle\right]+\mathrm{c} . \mathrm{c}\right\}
$$

with:

$$
\begin{aligned}
& \xi_{\mathrm{k}}=\frac{1}{2} \text { if } \mathrm{k}=0 \text { or } \mathrm{k}=\mathrm{m}+1 ; \xi_{\mathrm{k}}=1 \text { otherwise }, \mathrm{z}_{\mathrm{k}}=\exp \left(\frac{\mathrm{ik} \pi}{\mathrm{m}+1}\right), \quad\left|\psi\left(\mathrm{z}_{\mathrm{k}}, \mathrm{z}_{\mathrm{k}^{\prime}}\right)\right\rangle=\prod_{v>0}\left|\psi_{v}\left(\mathrm{z}_{\mathrm{k}}, \mathrm{z}_{\mathrm{k}^{\prime}}\right)\right\rangle \\
& \left.\left|\psi_{v}\left(\mathrm{z}_{\mathrm{k}}, \mathrm{z}_{\mathrm{k}^{\prime}}\right)\right\rangle=\mid \mathrm{B}_{1}^{v} \mathrm{z}_{\mathrm{k}} \mathrm{z}_{\mathrm{k}^{\prime}} \mathrm{A}_{v \mathrm{p}}^{+} \mathrm{A}_{v \mathrm{n}}^{+}+\mathrm{B}_{\mathrm{p}^{v}}^{v} \mathrm{z}_{\mathrm{k}^{\prime}} \mathrm{A}_{v \mathrm{p}}^{+}+\mathrm{B}_{\mathrm{n}}^{v} \mathrm{z}_{\mathrm{k}} \mathrm{A}_{v \mathrm{n}}^{+}+\mathrm{B}_{4}^{v} \sqrt{\mathrm{z}_{\mathrm{k}} \mathrm{z}_{\mathrm{k}^{\prime}}}\left(\mathrm{a}_{\tilde{v} \mathrm{p}}^{+} \mathrm{a}_{v \mathrm{n}}^{+}+\mathrm{a}_{\tilde{v} \mathrm{n}}^{+} \mathrm{a}_{v \mathrm{p}}^{+}\right)+\mathrm{B}_{5}^{v}\right\rfloor|0\rangle
\end{aligned}
$$

$\mathrm{m}, \mathrm{m}$ ' respectively refer to the projection order on the good neutron and proton numbers, c.c. means the complex conjugate with respect to $\mathrm{z}_{\mathrm{k}}$ and $\mathrm{C}_{\mathrm{mm}}$ is the normalization constant given by: 


$$
1=4(m+1)\left(m^{\prime}+1\right) C_{m m^{\prime}}^{2}\left\{\sum_{k=0}^{m+1} \sum_{k^{\prime}=0}^{m^{\prime}+1} \xi_{k} \xi_{k^{\prime}}\left[z_{k}^{-P_{n}} z_{k}^{-P_{p}} \prod_{j>0} A_{j}\left(z_{k}, z_{k^{\prime}}\right)+\bar{z}_{k}^{-P_{n}} z_{k}^{-P_{p}} \prod_{j>0} A_{j}\left(\bar{z}_{k}, z_{k^{\prime}}\right)\right]+c . c\right\}
$$

where we set:

$$
A_{j}\left(z_{k}, z_{k^{\prime}}\right)=z_{k} z_{k^{\prime}}\left(B_{1}^{j}\right)^{2}+z_{k^{\prime}}\left(B_{p}^{j}\right)^{2}+z_{k}\left(B_{n}^{j}\right)^{2}+2 \sqrt{z_{k} z_{k^{\prime}}}\left(B_{4}^{j}\right)^{2}+\left(B_{5}^{j}\right)^{2}
$$

In the second quantization and isospin formalism, the quadrupole moment operator reads:

$$
\hat{\mathrm{Q}}_{2}=\sum_{\mathrm{ij}} \mathrm{Q}_{\mathrm{ij}} \mathrm{a}_{\mathrm{ip}}^{+} \mathrm{a}_{\mathrm{jp}}
$$

where $\mathrm{Q}_{\mathrm{ij}}$ are the matrix elements of $\hat{\mathrm{Q}}_{2}$ evaluated in the single-particle basis.

Using the wave-function (4), i.e. before the projection, the expectation value of $\hat{\mathrm{Q}}_{2}$ may be written:

$$
\mathrm{Q}_{2 \mathrm{BCSnp}}=2 \sum_{\mathrm{i}>0} \mathrm{Q}_{\mathrm{ii}}\left[\left(\mathrm{B}_{1}^{\mathrm{i}}\right)^{2}+\left(\mathrm{B}_{\mathrm{p}}^{\mathrm{i}}\right)^{2}+\left(\mathrm{B}_{4}^{\mathrm{i}}\right)^{2}\right]
$$

After the projection, the expectation value of $\hat{\mathrm{Q}}_{2}$ is evaluated using the state (5), that is [10]:

$$
\begin{aligned}
& \mathrm{Q}_{2 \text { SBCSnp }}=8(\mathrm{~m}+1)\left(\mathrm{m}^{\prime}+1\right) \mathrm{C}_{\mathrm{mm}}^{2} \\
& \left\{\sum_{\mathrm{k}=0}^{\mathrm{m}+1} \sum_{\mathrm{k}^{\prime}=0}^{\mathrm{m}^{\prime}+1} \xi_{\mathrm{k}} \xi_{\mathrm{k}^{\prime}}\left[\mathrm{z}_{\mathrm{k}}^{-\mathrm{P}_{\mathrm{n}}} \mathrm{z}_{\mathrm{k}}^{-\mathrm{P}_{\mathrm{p}}} \sum_{\mathrm{i}>0} \mathrm{Q}_{\mathrm{ii}} \mathrm{D}_{\mathrm{i}}\left(\mathrm{z}_{\mathrm{k}}, \mathrm{z}_{\mathrm{k}^{\prime}}\right)+\overline{\mathrm{z}}_{\mathrm{k}}^{-\mathrm{P}_{\mathrm{n}}} \mathrm{z}_{\mathrm{k}}-\mathrm{P}_{\mathrm{p}} \sum_{\mathrm{i}>0} \mathrm{Q}_{\mathrm{ii}} \mathrm{D}_{\mathrm{i}}\left(\overline{\mathrm{z}}_{\mathrm{k}}, \mathrm{z}_{\mathrm{k}^{\prime}}\right)\right]+\mathrm{c} . \mathrm{c}\right\}
\end{aligned}
$$

where we set: $D_{i}\left(z_{k}, z_{k^{\prime}}\right)=\left[z_{k} z_{k^{\prime}}\left(B_{1}^{i}\right)^{2}+z_{k^{\prime}}\left(B_{p}^{i}\right)^{2}+\sqrt{z_{k} z_{k^{\prime}}}\left(B_{4}^{i}\right)^{2}\right] \prod_{\substack{j>0 \\ j \neq i}} A_{j}\left(z_{k}, z_{k^{\prime}}\right)$.

One may easily show that at the limit when the np pairing vanishes, Eqs. (7) and (8) reduce to their homologues in the pairing between like-particles case [5-6].

\section{Numerical results- Discussion}

The electric quadrupole moments of the ground-state of some even-even nuclei such as $0 \leq(\mathrm{N}-\mathrm{Z}) \leq 4$ have been calculated using the single-particle energies and eigen-states of a deformed Woods-Saxon mean-field. $\mathrm{Q}_{2}$ has been evaluated using four various approaches: in the pairing between like-particles case, before $\left(\mathrm{Q}_{2 \mathrm{BCS}}\right)$ and after the projection $\left(\mathrm{Q}_{2 \mathrm{SBCS}}\right)$, and in the $\mathrm{np}$ pairing case before $\left(\mathrm{Q}_{2 \mathrm{BCSnp}}\right)$ and after the projection $\left(\mathrm{Q}_{2 \mathrm{SBCSnp}}\right)$.

In order to evaluate the np pairing effect, before and after the projection, we introduced the relative discrepancies: $\delta \mathrm{Q}_{2}^{\mathrm{np}}=\left|\left(\mathrm{Q}_{2(\mathrm{~S}) \mathrm{BCSnp}}-\mathrm{Q}_{2(\mathrm{~S}) \mathrm{BCS}}\right) / \mathrm{Q}_{2(\mathrm{~S}) \mathrm{BCS}}\right|$. These quantities are given in the left part of Fig. 1 as a function of $\mathrm{Z}$ for various values of $(\mathrm{N}-\mathrm{Z})$. In the same way, we introduced the relative discrepancies: $\delta \mathrm{Q}_{2}^{\text {proj }}=\left|\left(\mathrm{Q}_{2 \mathrm{SBCS}(\mathrm{np})}-\mathrm{Q}_{2 \mathrm{BCS}(\mathrm{np})}\right) / \mathrm{Q}_{2 \mathrm{BCS}(\mathrm{np})}\right|$ in order to evaluate the projection effect, in the pairing between like-particles case and in the np pairing one. These quantities are given in the right part of Fig. 1 as a function of $\mathrm{Z}$ for various values of $(\mathrm{N}-\mathrm{Z})$. It then appears that the $\mathrm{np}$ pairing effect is not very important, either before or after the projection, since the relative discrepancy $\delta \mathrm{Q}_{2}^{\mathrm{np}}$ is at most $12 \%$. It also appears that this effect decreases as a function of (N-Z). Moreover, it seems that the np pairing effect is virtually the same before and after the projection.

On the other hand, one can see that the projection effect is somewhat small since the relative discrepancy $\delta \mathrm{Q}_{2}^{\text {proj }}$ is also at most $12 \%$. However, it is slightly less important in the np pairing case than when only the pairing between like-particles is considered. 

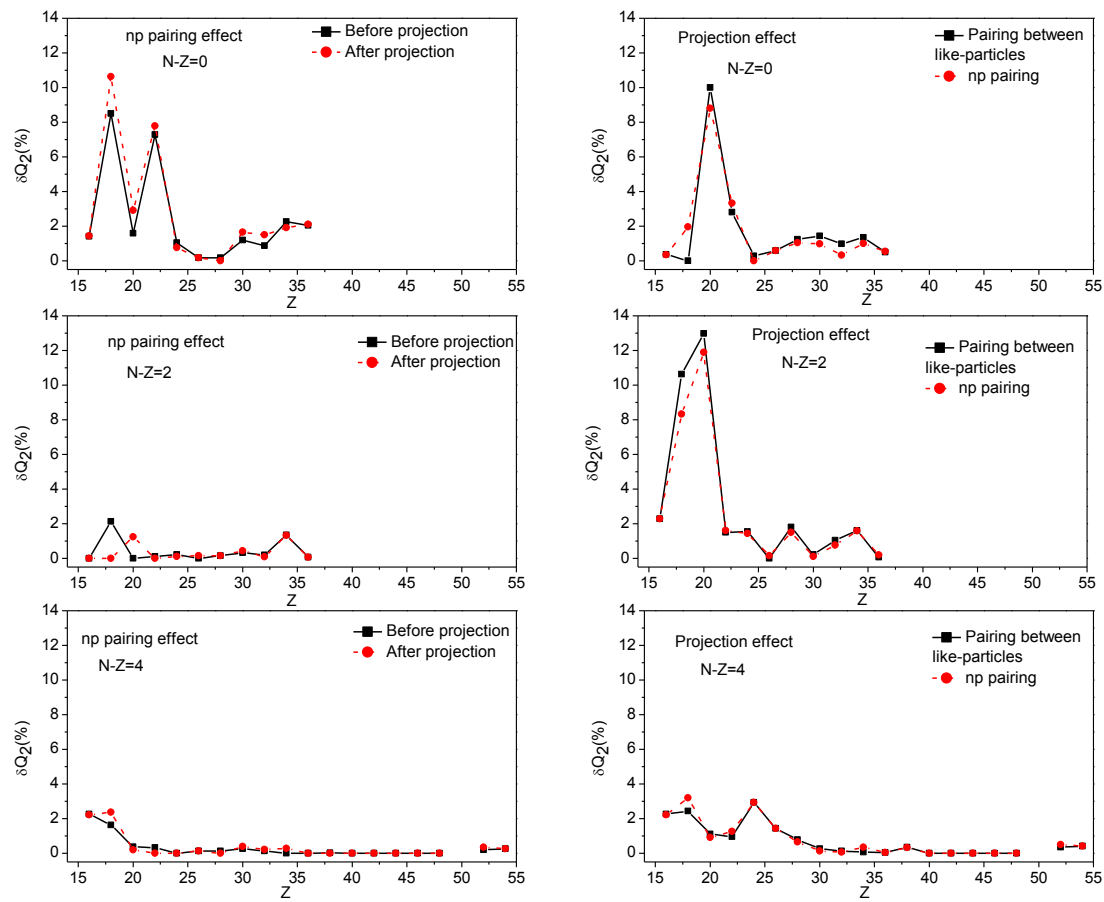

Figure 1. Study of the np pairing effect (at left) and the projection effect (at right) on the electric quadrupole moment (see the text for the notations), as a function of $\mathrm{Z}$ for various values of $(\mathrm{N}-\mathrm{Z})$.

\section{Conclusion}

An expression of the number-projected electric quadrupole moment $\mathrm{Q}_{2}$ has been established in the isovector pairing case using the SBCS projection method. The electric quadrupole moment of the ground-state of some even-even proton-rich nuclei has been calculated using the single-particle energies and eigen-states of a deformed Woods-Saxon mean-field. The obtained values have been compared to those obtained before the projection and when only the pairing between like-particles is considered. It has been shown that the np pairing effect is not very important, either before or after the projection, since the relative discrepancy does not exceed $12 \%$. However, this effect decreases as a function of $(\mathrm{N}-\mathrm{Z})$. In the same way, it has been shown that the projection effect is not very important and is roughly the same in the isovector pairing case and in the pairing between like-particles one.

\section{References}

1. B. Blank and M.J.G. Borge, Progr. Part. Nucl. Phys. 60, 403 (2008).

2. T. Faestermann, M. Gorska and H. Grawe, Prog. Part. Nucl. Phys. 69, 85 (2013).

3. A. L. Goodman, Phys. Rev. C 60, 014311 (1999).

4. G. Neyens, Rep. Prog. Phys. 66, 633 (2003).

5. N. Benhamouda, M.R. Oudih, N.H. Allal and M. Fellah, Nucl. Phys. A 690, 219 (2001).

6. N. Benhamouda, N.H. Allal, M. Fellah and M.R.Oudih, Int. J. Mod. Phys. E 14, 197 (2005).

7. D. Mokhtari, I. Ami, M. Fellah and N.H. Allal, Int. J. Mod. Phys. E 17, 655 (2008).

8. S. Kerrouchi, N.H. Allal, M. Fellah and M. Douici, Int. J. Mod. Phys. E 19, 1383 (2010).

9. M. Douici, N. H. Allal, M. Fellah, M.R. Oudih and N. Benhamouda, Int. J. Mod. Phys. E 21, 1250046 (2012).

10. M. Douici, N. H. Allal, M. Fellah, M.R. Oudih and N. Benhamouda, Int. J. Mod. Phys. E 22, 1350029 (2013). 